\title{
Advancing the DOHaD agenda in Africa
}

In 2016 the Health in Transition programme at the Stellenbosch Institute for Advanced Study (STIAS) established a long-term project on 'The Developmental Origins of Health and Disease and Sustainable Development Goals: Moving Towards Early Implementation'. The programme was predicated on the following:

1. The acceptance that Developmental Origins of Health and Disease $(\mathrm{DOHaD})$ theory had reached a point where it was possible to contemplate translation to health policy.

2. That Africa, which has the greatest health needs, also provided the greatest opportunities for improving health through improved health policies that emphasized early childhood development.

3. That the recently announced sustainable development goals (SDGs) ${ }^{1}$ provide us with an opportunity and energy, including potential financing, to do something more concrete and faster in relation to implementing some of the actionable outcomes of $\mathrm{DOHaD}$ thinking. Many of the SDGs, including \#1 (eliminate poverty), \#2 (eliminate hunger), \#3 (good health, which mentions chronic noncommunicable disease and also mental health) \#4 (quality education), \#5 (gender equity), \#6 (clean water and sanitation) and \#10 (reduced inequalities) are directly relatable to DOHAD.

In September 2016 STIAS hosted a workshop on DOHaD and SDGs. Resident Fellows and a number of African researchers and young scholars were invited to present their work. Some of those presenters agreed to add more data and reflection and submit scientific papers for publication in the Journal of Developmental Origins of Health and Disease. Another outcome of the workshop was agreement to establish an African Chapter of the International Society of Developmental Origins of Health and Disease, and this has now been accepted and endorsed by the DOHaD Council. At the same time there was agreement to establish a network of young African scientists working on DOHaD-related issues and, in collaboration with the African Academy of Science, this will be launched at a STIAS summer school in November 2017.

This focus issue of the Journal of Developmental Origins of Health and Disease celebrates the adoption of the African Chapter and includes six papers that address elements of the DoHAD agenda in the context of Africa. In their paper 'The Developmental Origins of Health and Disease and Sustainable Development Goals: mapping the way forward', Kajee et al. ${ }^{2}$ suggest that there is benefit to approaching together the implementation of two seemingly separate recent developments. First, on the global development agenda, we have the
United Nations General Assembly's 2015 finalized list of 17 SDGs. Several of the SDGs are related to health. Second, the field of $\mathrm{DOHaD}$ has garnered enough compelling evidence demonstrating that early exposures in life affect not only future health, but that the effects of that exposure can be transmitted across generations - necessitating that we begin to focus on prevention. The authors argue that implementing the 17 SDGs and $\mathrm{DOHaD}$ together will be beneficial in several ways, but will require attending to multiple, complex and multidisciplinary approaches as the point is reached for translating science to policy to impact. They also provide the context for working in this direction and make the case for a mutually reinforcing, synergistic approach to implementing SDGs and $\mathrm{DOHaD}$ in Africa.

Pregnant women and children under the age of 2 years have been an important target of $\mathrm{DOHaD}$ interventions, which have focused attention on 'the first thousand days of life'. ${ }^{3}$ In a paper 'Beyond the dyad: making $\mathrm{DOHaD}$ interventions more inclusive', Pentecost et $a l^{4}{ }^{4}$ acknowledge that while useful for rallying global attention, this 'thousand days' focus may inadvertently have deflected attention from other windows of opportunity, and argue for new interventions in future along two axes. First, that the focus should move beyond the current spectrum of girls, women and mothers to include boys, men and fathers and other family members; while being cognizant of the gender roles inherent in these framings, rather than focusing on individual parental action these frameworks should also encompass the wider social fields in which interventions are taking place rather than focussing on individual parental action. Second, that it will be beneficial to return to life-course approaches that frame interventions as part of longer-term strategies to provide the foundation for more comprehensive intervention strategies beyond the designated window of opportunity in early life. In essence, that there be a shift from a 'pre-conception' lens to a more holistic focus that addresses different stages of the life course, and discusses the importance and challenges of intervention design and inclusion of a more comprehensive audience for the $\mathrm{DOHaD}$ message.

A challenge for implementing DOHaD-defined health promotion for a more comprehensive audience is how to engage the 'at risk' population. This includes youth who traditionally are not a sub-set of the population who prioritize or even seriously consider their future health or that of their potential partners, let alone their offspring. But, Macnab and Mukisa ${ }^{5}$ report a school-based study conducted to identify the 'Priorities for African youth for engaging in the DOHAD agenda'. They conducted a survey in three 'Health Promoting Schools' (HPS) in rural Uganda. The WHO HPS model has 
had proven success engaging youth and providing education that improves health behaviours. $\mathrm{DOHaD}$ concepts were explained to pupils aged 12-15 years in discussion groups that are held regularly to promote dialogue on health issues. The pupils were then asked to discuss and prioritize what factors would make $\mathrm{DOHaD}$-related health promotion resonate with them, and what 'messages' and 'messengers' they would recommend to make learning about $\mathrm{DOHaD}$ most acceptable to youth. These are the first data from African youth charged with addressing how best to engage them in the $\mathrm{DOHaD}$ agenda.

Despite global and national strategies to reduce child malnutrition and death, millions of children under 5 years of age die every year, with two-thirds of the deaths being caused by preventable causes. Macharia et al. ${ }^{6}$ describe their study of 'The association between household food security and infant feeding practices in urban informal settings in Nairobi county, Kenya'. In a longitudinal design 1101 mother-child pairs were followed for 1 year to establish infant feeding practices and food security status of households. Most households were food insecure and hence had near similar feeding practices which indicates that mothers are not able to feed their children as recommended, and therefore there is a need to promote interventions that improve child feeding practices in this and similar poor resource settings.

Internationally there is evidence that antenatal depression has an intergenerational effect, however, there is a dearth of research from the African continent, and in the few studies which do exist depression tends to be examined alone, without looking at other comorbidities such as anxiety, stress or physiological illnesses, or vice versa, and most only examine mental health late in pregnancy. Redinger et al.' report on 'Antenatal depression and anxiety in the first trimester of pregnancy, in a high risk population in Soweto, South Africa'. This research was part of the Soweto 1000 Days Cohort study which was established to examine the independent and interrelated effects of multiple maternal factors that characterise populations with recent dramatic increases in obesity as well as persistent problems of HIV, psychosocial stress and micronutrient deficiencies. The authors have identified for the first time in African women that the risk of depression is high from early in pregnancy and this may have particular impacts on fetal development, birth outcomes and children's later development.

HIV-infected mothers face particular mental health risks, which in in turn impact their children's mental health, and population-based data from pregnancy and across the life course is needed in African settings is to improve prevention and treatment. Rochat $e t a l^{8}$ report the prevalence of psychological morbidity and factors associated with it in mothers during pregnancy in rural South Africa in their paper 'Psychological morbidity and parenting stress in mothers of primary school children by timing of acquisition of HIV infection: a longitudinal cohort study in rural South Africa'; those HIV positive and HIV negative were compared. Compared with being HIV negative, testing HIV positive in pregnancy doubled the odds of depression. Parenting stress was positively associated with acquisition of HIV after pregnancy and
HIV infection in pregnancy with later depression. The authors conclude that integrating parental support into treatment programmes across the life course, rather than in pregnancy alone, is important, as parenting stress can lead to dysfunctional parenting behaviours, is malleable to intervention, but is under-researched in Africa.

In conclusion, Africa faces significant and multiple health burdens (infectious and non-communicable diseases) and, therefore there needs to be a greater shift to prevention and setting up healthier trajectories across the life course. This is no easy task, but the implementation of a $\mathrm{DOHaD}$ life-course framework could see more African researchers contributing more to the field, as well as, offsetting such persisting intergenerational challenges such as stunting. To increase $\mathrm{DOHaD}$ research in Africa, we need to mobilize multi-sectoral partners (including science funders), utilize existing data and expertise on the continent, and foster a new generation of young African scientists engrossed in $\mathrm{DOHaD}$ science.

\section{References}

1. United Nations. Transforming our world: the 2020 Agenda for Sustainable Development. United Nations, New York, 2015. Retrieved 1 September 2017 from https://sustainabledevelopment. un.org/content/documents/212520302 Agenda for Sustainable Development web.pdf.

2. Kajee N, Sobngwi E, Macnab A, Daar AS. The Developmental Origins of Health and Disease and Sustainable Development Goals: mapping the way forward. J Dev Orig Health Dis. 2018; 9, 5-9.

3. International Society for Developmental Origins of Health and Disease. The Cape Town Manifesto - November 2015. International Society for Developmental Origins of Health and Disease, Cape Town, 2015. Retrieved 1 September 2017 from https://dohadsoc.org/wp-content/uploads/2015/11/DOHaDSociety-Manifesto-Nov-17-2015.pdf.

4. Pentecost M, Ross FC, Macnab A. Beyond the dyad: making Developmental Origins of Health and Disease (DOHaD) interventions more inclusive. J Dev Orig Health Disease. 2018; $9,10-14$.

5. Macnab AJ, Mukisa R. Priorities for African youth for engaging in DOHaD. J Dev Orig Health Disease. 2018; 9, 15-19.

6. Macharia T, Ochala S, Mutua MK, Kimani-Murage EW. Association between household food security and infant feeding practices in urban informal settlements in Nairobi, Kenya. J Dev Orig Health Dis. 2018; 9, 20-29.

7. Redinger S, Norris SA, Pearson RM, Richter L, Rochat T. First trimester antenatal depression and anxiety: prevalence and associated factors in an urban population in Soweto, South Africa. J Dev Orig Health Disease. 2018; 9, 30-40.

8. Rochat TJ, Houle B, Stein A, et al. Psychological morbidity and parenting stress in mothers of primary school children by timing of acquisition of HIV infection: a longitudinal cohort study in rural South Africa. J Dev Orig Health Disease. 2018; 9, 41-57.

9. Norris SA, Daar A, Balasubramanian, et al. Understanding and acting on the developmental origins of health and disease in Africa would lead to better health across generations. Glob Health Action. 2017; 10, 1334985, http://dx.doi.org/10.1080/16549716.2017. 1334985 . 
A. J. Macnab

Department of Pediatrics, University of British Columbia,

Vancouver, Canada;

Stellenbosch Institute for Advanced Study (STIAS),

Wallenberg Research Centre at Stellenbosch University,

Stellenbosch, South Africa

A. S. Daar

Dalla Lana School of Public Health and Department of Surgery,

University of Toronto, Toronto, Canada;

Stellenbosch Institute for Advanced Study (STIAS),

Wallenberg Research Centre at Stellenbosch University,

Stellenbosch, South Africa
S. Norris

MRC/Wits Developmental Pathways for Health Research Unit, Department of Paediatrics, Faculty of Health Sciences, University of the Witwatersrand, Johannesburg, South Africa; Stellenbosch Institute for Advanced Study (STIAS), Wallenberg Research Centre at Stellenbosch University, Stellenbosch, South Africa

$$
\text { J. C. Pauw }
$$

Stellenbosch Institute for Advanced Study (STIAS), Wallenberg Research Centre at Stellenbosch University, Stellenbosch, South Africa 